\title{
Influence of 1B Ischemic Ventricular Tissue on the Automaticity of Purkinje Fibers: A Simulation Study
}

\author{
E Ramírez ${ }^{1.2}$, J Sáiz ${ }^{2}$, B Trénor ${ }^{2}$, JM Ferrero (Jr $)^{2}$, \\ G Moltó $\dot{0}^{3}$ V Hernández ${ }^{3}$ \\ ${ }^{1}$ Departamento de Ingenierías, Instituto Tecnológico de Cuautla, Morelos México \\ ${ }^{2}$ Center for Research and Innovation in Bioengineering, Universidad Politécnica de Valencia, Spain \\ ${ }^{3}$ Grupo de Redes y Computación de Altas Prestaciones, Universidad Politécnica de Valencia, Spain
}

\begin{abstract}
A computer model has been developed to study the electrical activity of a Purkinje fiber of $1.5 \mathrm{~cm}$ coupled to a regionally $1 B$ ischemic ventricular fiber of $3 \mathrm{~cm}$ through a Purkinje-muscle resistance $\left(R_{p v j}\right)$. The ventricular fiber was composed of a $1 B$ ischemic zone, a border zone and a normal zone of $1 \mathrm{~cm}$ each. To simulate ischemia, we altered several electrophysiological parameters of the Luo-Rudy action potential model (Lrd00). The aim of this study was to investigate the influence of $1 B$ ischemic ventricular tissue on the automaticity of Purkinje cells and its role in ectopic activity that could provoke arrhythmias. Our study suggests that $1 B$ ischemic ventricular tissue enhances automaticity of Purkinje fibers and could have an important role on the generation of ectopic action potentials near the Purkinje-muscle junctions. This phenomenon may be involved in the generation of ectopic activity and cardiac arrhythmias.
\end{abstract}

\section{Introduction}

Sudden cardiac death is mainly due to ischemiainduced ventricular arrhythmias, in particular to ventricular fibrillation (VF) [1]. During acute ischemia, ventricular arrhythmias occur in two different phases. Firstly, arrhythmias take place during phase 1A, between 2 and 15 minutes after coronary occlusion. After an interval relatively free of arrhythmias, a second phase of arrhythmic events is observed (referred as 1B phase), between 15 and 60 minutes after the onset of ischemia [2]. It is known that during phase $1 \mathrm{~B}$, Purkinje fibers have an important role in the generation of arrhythmias.

Purkinje fibers adjacent to an ischemic region are proposed to play an important role in the genesis of ventricular arrhythmias during acute myocardial ischemia. Their relatively unstable resting membrane potential and their relatively long action potentials (APs) may predispose to the occurrence of cellular triggers (EADs and DADs) and automaticity [3, 4], which are known to be involved in arrhythmogenic processes.

During myocardial ischemia, a border zone located between normal and ischemic tissues has been experimentally observed. The existence of this zone nesting heterogeneities and important electrical alterations, might favor the occurrence of arrhythmias such as automatic activity, focal reexcitation or reentrant arrhythmias. The border zone is a cardiac region presenting inhomogeneous distribution of electrophysiological properties and has been associated with injury currents possibly responsible for arrhythmogenic processes [5].

Purkinje to ventricular conduction ( $\mathrm{P}-\mathrm{V}$ conduction) is considered to be an essential step in the cardiac excitation sequence. The Purkinje network is a thin layer of tissue coupled to the much thicker ventricular myocardium at discrete sites in the subendocardium referred as Purkinjeventricular junctions (PVJs). Purkinje-ventricular conduction is described as discontinuous because the conduction delay between the two regions is large (from 3 to $6 \mathrm{~ms})$, considering the relatively short distance $(0.1$ to $1.0 \mathrm{~mm}$ ) traveled by the pulse [6].

The aim of this study was to investigate the influence of 1B ischemic ventricular tissue on the automaticity of Purkinje cells and AP propagation, which have an important role in ectopic activity precursor for arrhythmias.

\section{Methods}

We simulated the electrical activity of a 1D Purkinje fiber of $1.5 \mathrm{~cm}$ coupled to a regional $1 \mathrm{~B}$ ischemic ventricular fiber of $3 \mathrm{~cm}$, through a Purkinje-muscle resistance $\left(\mathrm{R}_{\mathrm{PVJ}}\right)$, as depicted in figure 1 . The ventricular fiber comprised a 1B ischemic zone, a border zone and a normal zone of $1 \mathrm{~cm}$ each. 
DiFrancesco-Noble formulation was used to model the Purkinje ells AP [7]. The cells of Purkinje were considered cylindrical with a length of $150 \mu \mathrm{m}$ and a radio of $7.5 \mu \mathrm{m}$. Luo-Rudy model (LRd00) was used to calculate ventricular AP [8,9]. A finite-difference technique in space and time using the implicit method of Crank and Nicholson was used to solve the differential equation that describes AP propagation. Myocardial cells also were considered cylindrical with a length of $100 \mu \mathrm{m}$ and a radio of $11 \mu \mathrm{m}$.

To simulate ischemia, we altered several electrophysiological parameters of the Luo-Rudy action potential model (Lrd00) as proposed by Pollard and coworkers [10]. These parameters were linearly varied in the border zone from the normal values, in the normal tissue, to the ischemic values in the central ischemic zone as indicated in figure 1.

Purkinje cells autogenerated 10 pulses, which propagated through the whole fiber. The last autogenerated action potential was considered for analysis.

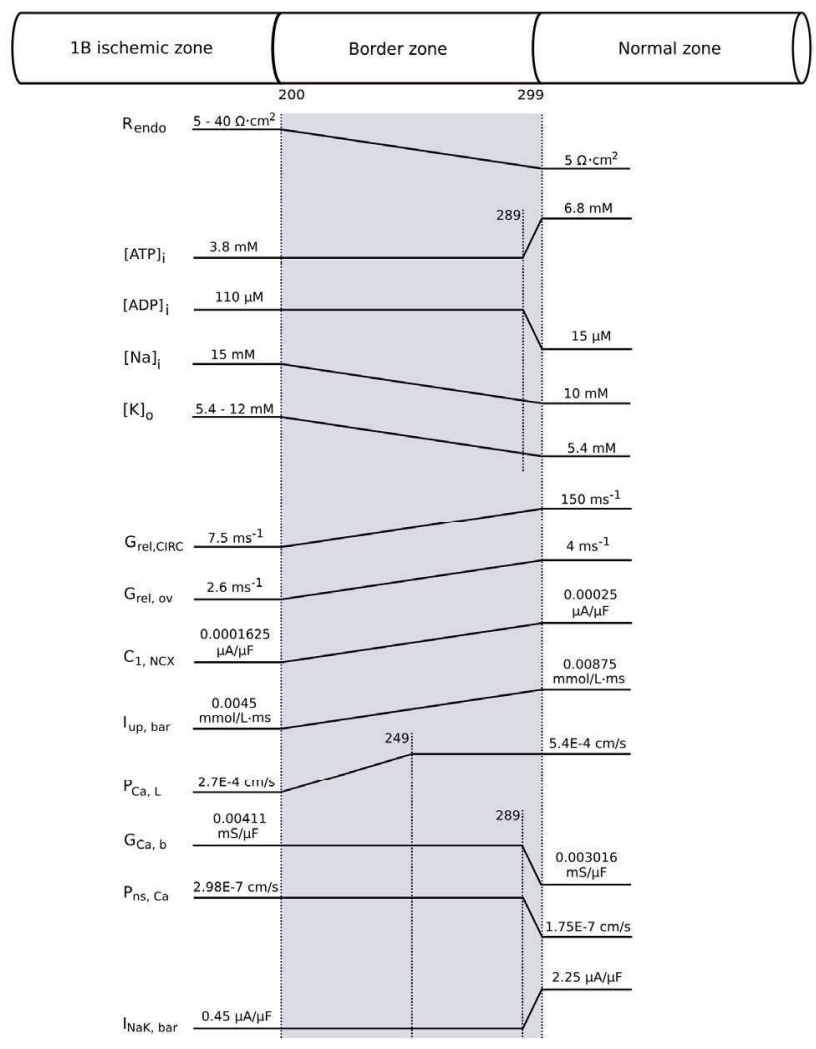

Figure 1. One-dimensional model of Purkinje fiber coupled to $1 \mathrm{~B}$ ischemic ventricular tissue. The values of different parameters were linearly varied, in the border zone, from their normal values (in normal zone) to their ischemic values.

\section{Results and Discussion}

In this study, the effects of ventricular 1B ischemia on the electrical properties of Purkinje fibers were analyzed. In first instance, we simulated AP propagation in Purkinje fiber coupled to normal ventricular fiber. Under such circumstances, the basic cycle length (BCL) of the autogenerated pulse in Purkinje fiber yielded $1659 \mathrm{~ms}$, as indicated in table 1. In this table, we show the changes of BCL in Purkinje fibers (third column 3) for different extracellular potassium concentrations $\left(\left[\mathrm{K}^{+}\right]_{\mathrm{o}}\right)$ in the ischemic zone (first column) and the site where the impulse is generated in the Purkinje fiber (second column). It is to be noted that the first row corresponds to normal myocardium and the following rows correspond to ischemic conditions with $\mathrm{R}_{\mathrm{PVJ}}=16 \Omega \cdot \mathrm{cm}^{2}, \mathrm{R}_{\text {endo }}=5$ $\Omega \cdot \mathrm{cm}^{2}$. We observed that, when the ventricle was rendered ischemic, normal Purkinje automaticity was altered, as BCL decreased. This effect could have important implications in arrhythmogenesis as have been hypothesized by Zipes and Antzelevitch [10,11].

Also Verkerk and coworkers highlighted the important role of ischemia $1 \mathrm{~B}$ in the alteration of electrical properties in Purkinje fibers [4].

In analyzing the role of hyperkalemia we observed that the more hyperkalemic was ischemic tissue, the lesser BCL was recorded in Purkinje fiber, reaching $1518 \mathrm{~ms}$ for $\left[\mathrm{K}^{+}\right]_{\mathrm{o}}=12 \mathrm{mmol} / \mathrm{L}$. This reduction represents a change of $8.5 \%$ with respect to normal conditions. Indeed, potassium accumulation is known to be pivotal in electrical alterations related to arrhythmogenic processes [12].

\begin{tabular}{|c|c|c|}
\hline $\begin{array}{c}{\left[\mathrm{K}^{+}\right]_{\mathrm{o}}} \\
(\mathrm{mmol} / \mathrm{L})\end{array}$ & $\begin{array}{c}\text { Origin of impulse } \\
(\text { cell } \#)\end{array}$ & $\begin{array}{c}\text { BCL } \\
(\mathrm{ms})\end{array}$ \\
\hline 5.4 & 2 & 1659 \\
\hline 6 & 2 & 1657 \\
\hline 7 & 2 & 1647 \\
\hline 8 & 2 & 1638 \\
\hline 9 & 2 & 1626 \\
\hline 10 & 54 & 1607 \\
\hline 11 & 72 & 1570 \\
\hline 12 & 80 & 1518 \\
\hline
\end{tabular}

Table 1. Effect of $\left[\mathrm{K}^{+}\right]_{0}$ in $1 \mathrm{~B}$ ischemic ventricular fiber on the site and BCL of the autogenerated action potential in Purkinje fiber.

Another observed phenomenon was the fact that the site of generation of the autogenerated pulse along the Purkinje fiber changed with hyperkalemic conditions. In normoxia, the autogenerated pulse was initiated in the left edge of the Purkinje fiber, whereas an increase in $\left[\mathrm{K}^{+}\right]_{\mathrm{o}}$ up to $12 \mathrm{mmol} / \mathrm{L}$, changed the site of generation of the pulse to a closer site to Purkinje-muscle junction (PMJ). 
This effect is depicted in figure 2, where action potentials obtained in Purkinje fiber and in the $1 \mathrm{~B}$ ischemic zone are shown. It can be observed that the pulse was autogenerated in cell \#69 in the Purkinje fiber. The elicited action potential propagated then in both directions, forwards to ventricular cells and backwards to the rest of Purkinje cells as indicated by the arrows.

This figure also shows the difference in propagation velocity in Purkinje fiber and ventricular fiber, as documented by other authors $[13,14]$. Indeed the delay in propagation is greater in $1 \mathrm{~B}$ ischemic zone.

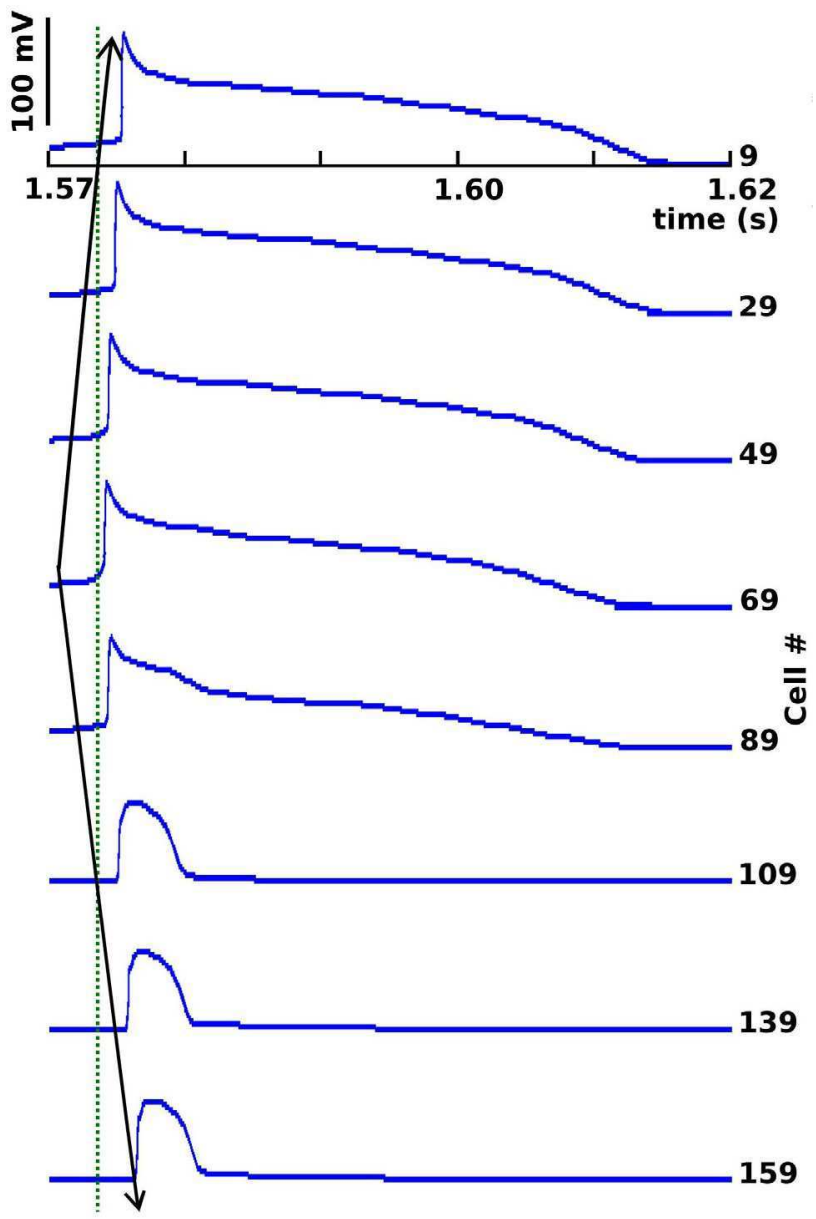

Figure 2. Action potentials in several cells of Purkinje fibers and the 1B ischemic zone. The arrows show the propagation direction of the impulse autogenerated in cell \#69.

Another important result of the present study was the analysis of AP propagation and block through the coupling of purkinje fibers and $1 \mathrm{~B}$ ischemic myocardium. In figure $3 \mathrm{~A}$, we show in different colors the APs obtained in the midle of Purkinje fiber, in 1B ischemic zone, in the border zone and in the normal zone of the ventricular fiber, for hyperkalemic conditions of $\left[\mathrm{K}^{+}\right]_{\mathrm{o}}=$ $11.5 \mathrm{mmol} / \mathrm{L}$ in the central ischemic zone. In this case,
AP propagated through the Purkinje fiber and the myocardial cells. An increase in $\left[\mathrm{K}^{+}\right]_{\mathrm{o}}$ up to $11.7 \mathrm{mmol} / \mathrm{L}$, provoked changes in propagation. Indeed, although AP could propagate through Purkinje fiber, propagation was blocked in the ventricular fiber, as shown in figure 3B. This figure represents the variation of the safety factor (SF) along the fiber. The SF was used to quantify the propagation safety in the fibers of the model. A value under the unity of the SF indicates the occurrence of propagation block. We estimated the SF basing our calculations on the formulation proposed by Shaw and Rudy and modified by Romero et al. $[15,16]$. In the Purkinje fiber, the SF yielded 2.6 and in the ventricular fiber this parameter varied in the different zones. The SF in the normal zone had a value of 1.9, similar to the value calculated by Shaw and Rudy and Romero et al. in normal conditions as indicator of safety in AP propagation $[15,16]$. The peak observed in the figure was related to the autogeneration of the impulse in the Purkinje fiber. As we previously mentioned, the site of generation of the pulse depended on the conditions of 1B ischemia and mainly on hyperkalemia.
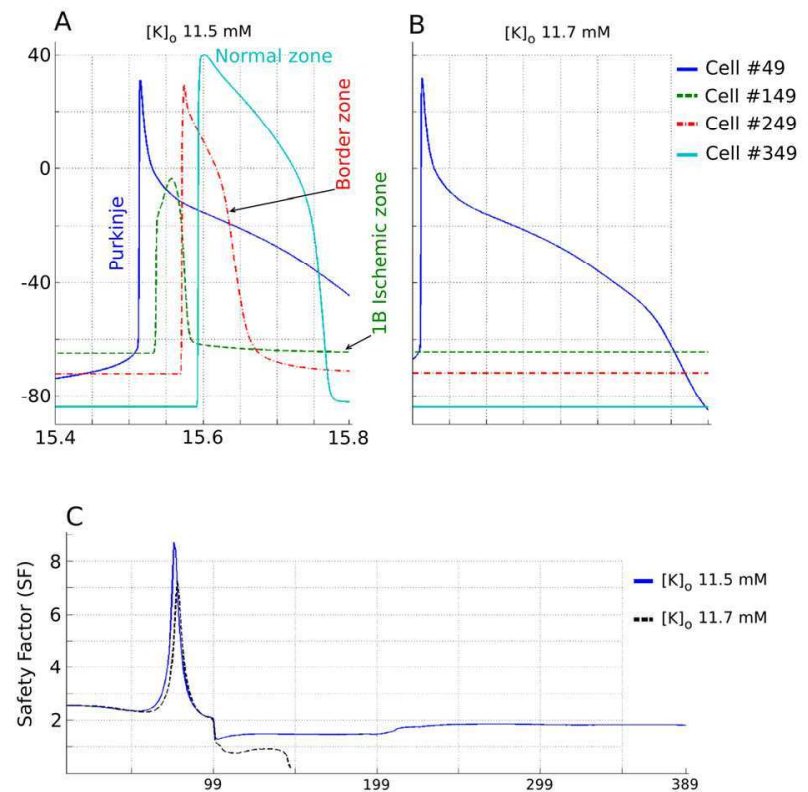

Figure 3. A, B) AP Propagation and block of in the fibers of the model. The APs were obtained in the middle of the fibers. C) Estimated safety factor along the fiber of the model.

\section{Conclusions}

The simulation of our study shows that $1 \mathrm{~B}$ ischemic ventricular tissue enhances automaticity of Purkinje fibers and could have an important role on the generation of ectopic action potentials near the Purkinje-muscle junctions. This phenomenon may be involved in the 
generation of ectopic activity and cardiac arrhythmias. We also observed the important role of hyperkalemia present in ischemic 1B tissue in AP propagation through purkinje-ventricle junction.

\section{Acknowledgements}

This work has been partially supported by the Plan Nacional de Investigación Científica, Desarrollo e Innovación Tecnológica del Ministerio de Educación y Ciencia of Spain (TEC2005-04199).

\section{References}

[1] De Groot JR, Wilms-Schopman FJ, Opthof T, Remme CA and Coronel R. Late ventricular arrhythmias during acute regional ischemia in the isolated blood perfused pig heart. Role of electrical cellular coupling. Cardiovascular Research 2001; 50:362-372.

[2] Kaplinsky E, Ogawa S, Balke CW and Dreifus LS. Two periods of early ventricular arrhythmia in the canine acute myocardial infarction model. Circulation 1979; 60:397403.

[3] Arnar DO and Martins JB. Purkinje involvement in arrhythmias after coronary artery reperfusion. Am J Physiol Heart Circ Physiol 2002; 282: H1189-H1196.

[4] Verkerk AO, Veldkamp MW, Coronel R, Wilders R and van Ginneken ACG. Effects of cell-to-cell uncoupling and catecholamines on Purkinje and ventricular action potentials: implications for phase- $1 \mathrm{~b}$ arrhythmias. Cardiovascular Research 2001; 51: 30-40.

[5] Picard S, Rene Rouet R, Ducouret P, Puddu PE, Flais F, Criniti A, Monti F and Gerard JL. KATP channels and 'border zone' arrhythmias: role of the repolarization dispersion between normal and ischaemic ventricular regions. British Journal of Pharmacology 1999;127:16871695

[6] DiFrancesco D and Noble D. A model of cardiac electrical activity incorporating ionic pumps and concentration changes. Philos Trans R Soc Lond B Biol Sci 1985; 307: 353-398.

[7] Luo CH, Rudy Y. A dynamic model of the cardiac ventricular action potential: I. Simulations of ionic currents and concentration changes. Circulation Research 1994; 74:1071-1096.
[8]

[9] Faber GM, Rudy Y. Action potential and contractibility changes in $[\mathrm{Na}(+)](\mathrm{i})$ overloaded cardiac myocytes: a simulation study. Biophysics Journal 2000; 78:2392-2404.

[10] Pollard A, Cascio W, Fast V and Knisley S. Modulation of triggered activity by uncoupling in the ischemic border. A model study with phase $1 \mathrm{~b}$-like conditions. Cardiovase Res 2002; 56:381-392.

[11] Zipes DP. Mechanisms of clinical arrhythmias. J Cardiovasc Electrophysiol 2002; 14:902-912.

[12] Antzelevitch C. Basic mechanisms of reentrant arrhythmias. Curr Opin Cardiol 2001; 16:1-7.

[13] Rodriguez-Sinovas, A. y Cinca, J. (1999). [Sudden death (II). Myocardial ischemia and ventricular arrhythmias in experimental models: triggering mechanisms], Rev Esp Cardiol 1999; 52:851-859.

[14] K. Walton MK and Fozzard HA. Experimental study of the conducted action potential in cardiac Purkinje strands. Biophysical Journal 1983; 44:1-8.

[15] Rohr S. Role of gap junctions in the propagation of the cardiac action potential. Cardiovascular Research 2004; 62: $309-322$

[16] Shaw R. and Rudy Y. Ionic mechanisms of propagation in cardiac tissue. Roles of the sodium and L-type calcium currents during reduced excitability and decreased gap junction coupling. Circ Res 1997; 81: 727-41.

[17] Romero L, Trenor B, Ferrero (Jr) JM and Saiz J. A sensitivity study of the safety factor for conduction in the myocardium. Computers in Cardiology 2005; Vol. 32:873876.

Address for correspondence

Esteban Benito Ramírez Islas

Departamento de Ingenierías.

Instituto Tecnológico de Cuautla.

Libramiento Cuautla-Oaxaca S/N, Cuautla Morelos, México. esrais@doctor.upv.es 